\title{
Crystal structure of Senecavirus A 3C protease
}

\author{
Kaiwen Meng*, Lijie Zhang*, Geng Meng† \\ a College of Veterinary Medicine, China Agricultural University, 100193, Beijing, China \\ * These authors contribute equally to this work. \\ $\uparrow$ Corresponding author. \\ E-mail address:mg@cau.edu.cn
}

\begin{abstract}
Senecavirus A (SVA), an emerging picornavirus in porcine population, could infect porcines of all age group and cause FMD-like symptoms. Picornaviridae, a group of RNA viruses do harm to both human and stocks; however, most of picornaviruses are lack of effective vaccines and drugs. Picornaviral $3 \mathrm{C}$ protease $\left(3 \mathrm{C}^{\mathrm{pro}}\right)$, as an important role in virus maturation, they basically take charge of poly-protein cleavaging, RNA replication, and multiple interventions on host cells. In this study, we successfully solved the crystal structure of $3 \mathrm{C}^{\text {pro }}$ at $1.9 \AA$ resolution. The results showed several differences of the binding groove within picornaviral $3 \mathrm{C}^{\mathrm{pro}}$, and prompted that the accommodate ability of the pocket may associate with the cleavage efficiency. The further research on $3 \mathrm{C}^{\text {pro }}$ cleavage efficiency based on structural biology, will prospectively provide an instruction on designing of efficient $3 \mathrm{C}^{\mathrm{pro}}$ for universally proteolysis in picornaviral VLP production.
\end{abstract}

Key word: Senecavirus A, 3C protease, X-ray diffraction, crystal structure 


\section{Introduction}

Senecavirus A (SVA), once called Seneca Valley Virus (SVV), is an emerging virus in porcine population that has been popular worldwide since 2015. SVA could infect porcine of all age group, clinically manifest symptoms indistinguishable from those of foot-and-mouth disease (FMD) (Zhang et al., 2018).

SVA belongs to the genus Senecavirus, family Picornaviridae. The virus genome consists of a positive sense, single-stranded RNA of $\sim 7280 \mathrm{nt}$ in length, encoding a large precursor poly-protein. $3 \mathrm{C}$ protease $\left(3 \mathrm{C}^{\mathrm{pro}}\right)$, a chymotrypsin-like cysteine protease shared by all Picornavirus, plays an important role in virus maturation, they basically take charge of poly-protein's proteolytic processing. Ten of the thirteen cleavage sites of the poly-protein are carried out by the $3 \mathrm{C}$ protease $\left(3 \mathrm{C}^{\mathrm{pro}}\right.$ ), including VP2 / VP3, VP3 / VP1, VP1 / 2A, 2B / 2C, 2C / 3A, 3A / 3B1, 3B1 / 3B2, 3B2 / $3 \mathrm{~B} 3,3 \mathrm{~B} 3 / 3 \mathrm{C}$ and 3C / 3D. According to Berger and Schechter's nomenclature (Berger and Schechter, 1970), the residues within the substrate preceding and following the cleavage site is denoted $\mathrm{P}$ and $\mathrm{P}$ ', respectively; and those subsites within the $3 \mathrm{C}^{\text {pro }}$ that accommodate $\mathrm{P}$ or $\mathrm{P}$ ' residues are numbered as $\mathrm{S}$ and $\mathrm{S}^{\prime}$. Most picornaviral $3 \mathrm{C}^{\mathrm{pro}}$ exhibit marked preference for $\mathrm{P} 1$, and P1' residue types (Gln-Gly junctions), whereas the rest of the P and P' positions show less sequence conservation. Surprisingly, mutations at those less conservative positions would dramatically influence the rate of peptide cleavage by $3 C^{\text {pro }}$ (Lu et al., 2011).

As Picornavirus basically rely on $3 C^{\text {pro }}$ to generate the individual structural and nonstructural proteins, $3 \mathrm{C}^{\text {pro }}$ is a necessary component for Picornaviral virus-like particle (VLP) production. However, the limitation of the use of $3 \mathrm{C}^{\text {pro }}$ is considerably significant: the tolerance of expression system against protease activity, the proportion between structural protein and $3 \mathrm{C}^{\text {pro }}$ expression, and the cleavage efficiency issue (Belsham and Bøtner, 2015; Polacek et al., 2013; Porta et al., 2013).

There are already a lot of work reveals the structural basis for $3 C^{\text {pro, }}$ s substrate recognition, as well as the relationship between proteolytic efficiency and cleavage site mutations. We believed that the structural information of $3 C^{\text {pro }}$ will offer an instruction to us to design efficient $3 C^{\text {pro }}$ for universally proteolysis in picornaviral VLP production. 


\section{Results \\ Phylogenetics and cleavage specificities of SVA $3 C^{\text {pro }}$}

Phylogenetic comparison of the sequence of SVA $3 \mathrm{C}^{\mathrm{pro}}$ with those of other picornaviruses suggests that SVA is related closely to Encephalomyocarditis virus (EMCV) and Human TMEV-like cardiovirus (HTCV), and more closely to Foot-and-mouth disease virus (FMDV) among all structure solved picornaviral $3 \mathrm{C}^{\text {pro }}$ (Fig.1).

We carried out an investigation on the sequence variation of the cleavage site associate with structural proteins (Fig. 2). The four distinct structural proteins of SVA are termed VP1-4, which are responsible for inducing humoral and cellular immunity in the animal body. As other picornavirus, the SVA $3 C^{\text {pro }}$ can specifically cleave P1 region into VP0, VP1 and VP3. VP0 will be further cleavaged into VP2 and VP4 during the encapsidation of RNA by unknown mechanism(Belsham and Bøtner, 2015). The cleavage site of SVA between VP0-VP3 is Q/G, which is consistent with the cleavage site of most picornaviruses. Whereas the cleavage site between VP1-VP3 is $\mathrm{H} / \mathrm{S}$, which is uncommon.

Alignment of peptides spanning four residues either side corresponding to VP0-VP3 and VP1-VP3 cleavage positions, reveals that picornaviral $3 \mathrm{C}^{\text {pro }}$ share similar substrate specificity in recognizing small hydrophobic amino acid residues at most positions, except P1 and P2. The P1 position is invariably occupied by hydrophilic amino acid, and in most cases it is occupied by a glutamine. On the contrary, $\mathrm{P} 2$ position shows no significant preference on amino acid residues, whether it is acidic, basic, aliphatic or polar.

However, the preference for small hydrophobic residues on most position is not an absolute. For SVA, the P4 position in VP0-VP3 and VP1-VP3 cleavage sequence were strikingly occupied by bigger amino acid residues, aspartic acid and tyrosine respectively, instead of alanine and proline in most cases. For other picornaviruses, seen in Fig. 2, amino acid residue with bigger side chain and/or different property also can be accommodate by a same subsite, that makes the $3 \mathrm{C}^{\text {pro }}$ possible to recognize and cleave different site within the poly-protein. But this tolerance also brings notable variety in cleavage efficiency(Birtley et al., 2005; Lu et al., 2011; Zunszain et al., 2010). This phenomenon can be explained by the subsites' structure and will be discussed later.

\section{Overall structure of SVA $3 C^{\text {pro }}$}

The $3 \mathrm{C}^{\text {pro }}$ exists as a monomer in solution, as indicated by gel filtration (not shown). Crystals grew from $0.18 \mathrm{M}$ lithium chloride ( $\mathrm{pH} 7.0$ ), 12 to $18 \%$ PEG 3350. The crystal structure of SVA $3 \mathrm{C}^{\mathrm{pro}}$ was determined at $1.9 \AA$ resolution by the molecular replacement. The final refinement of the structure generated the R/Rfree factors of $18.43 / 23.55 \%$ (Table 1). The crystal belongs to space group P $122_{1} 1$, with two $3 \mathrm{C}^{\text {pro }}$ monomers per asymmetric unit. The two molecules are very similar, and the root mean square deviation (RMSD) for all of the C $\alpha$ atoms in two molecules was $0.258 \AA$. Therefore, unless otherwise noted, in comparisons below we shall be referring to one molecule in the asymmetric unit.

SVA $3 \mathrm{C}^{\text {pro }}$ adopts a typical chymotrypsin fold that is similar to those of other picornaviral $3 \mathrm{C}^{\text {pro }}$. The overall structure of SVA $3 C^{\text {pro }}$ is shown in Fig. 3. It contains 204 amino acids from residues D3 to R206, forming two domains. The first domain is largely composed of a 7 -stranded $\beta$-barrel structure (A1 to G1). The second domain also contains a compact barrel core, which is composed of $8 \beta$-strands (A2 to H2) arranged in an antiparallel manner. Overall, the two domains are connected via a long loop (amino acids 90 to $113 \mathrm{aa}$ ) over the "rear" surface of the molecule. The 
catalytic triad of His 48, Asn 84, Cys 160 is located in the cleft formed by the two $\beta$-barrel domains.

\section{Comparison of SVA $3 C^{\text {pro }}$ and related picornaviral protease}

Although they belong to the same family, the multi-sequences alignment shows that SVA $3 C^{\text {pro }}$ only shares $\leq 40 \%$ amino acid sequence identity with other picornaviral $3 \mathrm{C}^{\text {pro }}$ (Fig. 4), among which $3 \mathrm{C}^{\text {pro }}$ of EMCV shares the highest sequence similarity of $37.36 \%$, while EV71 $3 \mathrm{C}^{\text {pro }}$ shares the lowest similarity, $15.8 \%$. In addition, FMDV Type A $3 \mathrm{C}^{\text {pro }}$ also shows as high as $23.86 \%$ sequence align percent identity with SVA $3 \mathrm{C}^{\mathrm{pro}}$, which is the highest among all structure solved picornaviral $3 \mathrm{C}^{\text {pro }}$. Only three amino acid residues are invariable among all reference sequences, including H48, G158 and G161. H48 is one of the catalytic residue, and the latter two are associated with catalytic important motif G-X-C/S-G-G. Besides, G176, H178, G181 located on G2 strand are also highly conserved. While SVA $3 \mathrm{C}^{\mathrm{pro}}$ possess a relatively conservative catalytic motif as other picornaviral $3 C^{\text {pro }}$, it doesn't have a characteristic KFRDI motif. The KFRDI motif was previously characterized as one of RNA binding motifs in picornaviral $3 \mathrm{C}^{\text {pro }}$, it is mutated into SFPNN (95-99aa) in SVA 3C ${ }^{\text {pro }}$ (Leong et al., 1993; Matthews et al., 1994; Mosimann et al., 1997; Walker et al., 1995).

Overall, despite SVA $3 C^{\text {pro }}$ shares low homology with other picornaviral $3 C^{\text {pro }}$, SVA $3 C^{\text {pro }}$ still maintains the classic chymotrypsin fold. Superposition of the SVA $3 \mathrm{C}^{\text {pro }}$ structure with the other solved picornaviral $3 \mathrm{C}^{\mathrm{pro}}$ structures resulted in a root mean square difference (RMSD) in $\alpha$-carbon positions about 1.6-1.9 $\AA$ (Table 2). Here we focus on five positions with sequence insertion, which resulting in structural differences in SVA $3 \mathrm{C}^{\text {pro }}$ (Fig. 5).

The first sequence insertion occurs between 24-30aa. With six more amino acid residues, SVA $3 \mathrm{C}^{\text {pro }}$ contains a longer loop between $\beta A 1-\mathrm{B} 1$ strands further away from the substrate binding groove than PV(1L1N, 4DCD), CVA16(3SJ8, 3SJ9), CVB3(2ZU1, 2ZU3), EV68(3ZV8, 3ZVA) and EV71 (3OSY, 3SJO). The superposition consequence shows that short loop between $\beta \mathrm{A} 1-\beta \mathrm{B} 1$ strands will only adopt a slight change on angle after substrate combination. On the contrast, the longer loops of the $3 \mathrm{C}^{\text {pro }}$ from FMDV-A(2BHG, 2WV5), FMDV-SAT2(5HM2) and HAV(1HAV, $2 \mathrm{HAL}$ ) resulting from sequence insertion shows significant transformation after combining substrate. In FMDV $3 \mathrm{C}^{\text {pro }}$-peptide complex, the loop between $\beta A 1-\mathrm{B} 1$ strands bend away from the cleft; and in HAV $3 \mathrm{C}^{\text {pro }}$-inhibitor complex, the loop move toward the binding groove. Considering about 24-30aa are associate with the recognition of the $\mathrm{P}$ ' portion of substrate, these result implies that the $\beta A 1-B 1$ strands of SVA $3 C^{\text {pro }}$ might be a flexible structure.

Locate into the structure, the $\alpha 2$ helix to $\beta F 1$ strand segment possibly participates in forming the side wall and floor of the S' pocket. Residues insertion between 54-57aa and 66-71 aa in SVA 3C ${ }^{\text {pro }}$ resulting in a longer $\alpha 2$ helix, which draws the loop between $\alpha 2$ helix and $\beta \mathrm{D} 1$ strand near to $\beta A 1-B 1$ strands, meanwhile $\beta D 1-E 1$ strands bends away from the protein surface. The continuous $\beta E 1$ strand in the $3 C^{\text {pro }}$ from PV, CVA, CVB, EV68 and EV71 is broken into $\beta E 1$ and $\beta F 1$ in SVA, FMDV and HAV. A short loop protruding from the outer surface is formed between $\beta E 1$ and $\beta F 1$ in SVA $3 C^{\text {pro }}$, while in the $3 \mathrm{C}^{\text {pro }}$ from FMDV and HAV a short helix located in that position. In the $\alpha 2$ helix to $\beta E 1$ strand segments, SVA $3 C^{\text {pro }}$ shares similar structure with FMDV $3 C^{\text {pro }}$. However, $\mathrm{HAV} 3 \mathrm{C}^{\text {pro }}$ is less structure conserved between $\alpha 2$ helix and $\beta E 1$ strand when compared with SVA $3 \mathrm{C}^{\text {pro }}$ and FMDV $3 \mathrm{C}^{\text {pro }}$ : a shorter $\alpha 2$ helix followed by a loop with similar orientation of PV, CVA, CVB, EV68 and EV71; an extra helix between $\alpha 2$ helix and $\beta$ D1 strand; and a longer $\beta D 1-E 1$ 
strand.

The flexible surface loop between $\beta \mathrm{C} 2$ and $\beta \mathrm{D} 2$ strands, denoted as $\beta$-ribbon, whose configuration is not affected by the sequence insertion between 133-138aa. The $\beta$-ribbon plays an important role in recognizing the P2-P4 region of peptide substrates by transforming between two conformations ("open" and "close"), the mobility of the $\beta$-ribbon is relevant to protease activity(Cui et al., 2011). SVA $3 C^{\text {pro }}$ in this study retain this $\beta$-ribbon in a close state, i.e. the loop is located over the substrate binding groove with its apical tip pointing toward the protease active site.

The last sequence insertion is observed between 170-174aa. Thus, SVA $3 C^{\text {pro }}$ has a longer $\beta F 2-G 2$ strands orient differ from PV, CVA, CVB, EV68 and EV71. In PV, CVA, CVB, EV68 and EV71, $\beta$ F2-G2 strands in $3 C^{\text {pro }}$ bent toward $\alpha 1$ helix. The FMDV and HAV shares similar $\beta F 2-G 2$ strand with SVA $3 \mathrm{C}^{\text {pro }}$, albeit a short helix was formed on the tip in HAV $3 \mathrm{C}^{\text {pro }}$.

In general, both sequence and structural alignments indicate that SVA $3 \mathrm{C}^{\text {pro }}$ has a higher variability in the $\mathrm{N}$-terminal domain compared with other known picornaviral $3 \mathrm{C}^{\mathrm{pro}}$, while the C-terminus is relatively conservative. Furthermore, piles of researches were focused on $\mathrm{C}$ terminus and revealed that the $\mathrm{C}$-terminus plays an important role in substrate recognition.

\section{Prediction of the subsite conformation in SVA $3 C^{\text {pro }}$}

In order to identify residues of SVA $3 \mathrm{C}^{\text {pro }}$ directly involve in substrate binding, we superimpose the SVA $3 C^{\text {pro }}$ onto the FMDV $3 C^{\text {pro }}$-APAKELLNF peptide co-crystal structure, CVA

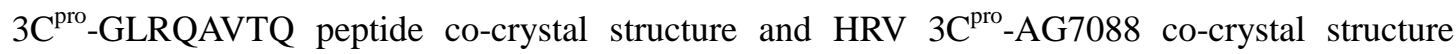
(Fig.6). The width of S subsite within SVA $3 \mathrm{C}^{\text {pro }}$ and FMDV $3 \mathrm{C}^{\mathrm{pro}}$ are shown in table 3.

In picornavirus, $\mathrm{P} 4$ residue exhibits conservatism, whose favored amino acid is valine, proline and alanine. However, as mentioned before, the P4 position in VP0-VP3 and VP1-VP3 cleavage sequence of SVA are occupied by amino acid residues with larger side chain, i.e. aspartic acid and tyrosine. This phenomenon can be explained by the conformation of the S4 subsite. In SVA 3C $\mathrm{C}^{\text {pro }}$, the S4 subsite pocket wall is formed by 135-141aa and 182-186aa. Among them, T139, S140, D141 and A182 may interact with P4 residue according to the superposition result. It is noteworthy that in SVA $3 \mathrm{C}^{\mathrm{pro}}$, these positions are variable and further away from the center of the substrate binding groove than the other structures in the reference. Thus, the wider S4 subsite might be a clue that residues larger than valine or proline can be tolerate by the protease. As for the tolerance of lager side chain in limited S4 subsite, e.g. the S4 position of HRV is limited but able to accommodate an asparagine (Fig. 2A), also can be explained by these interacted positions: an H-bond formed by peptide backbone atoms only, suggesting a degree of side-chain variation at these positions is acceptable.

Many structure solved picornaviral 3C ${ }^{\text {pro }}$ haven't shown significant S3 pocket(Curry et al., 2007; Zunszain et al., 2010). The superimpose result indicate A180 or G181 within SVA 3C $\mathrm{C}^{\text {pro }}$ may contact $\mathrm{P} 3$ with backbone $\mathrm{H}$-bond. And this positions are highly conserved in picornavirus.

As previously noted, the $\mathrm{P} 2$ residues of the picornaviral substrate peptide are variable. The $\mathrm{S} 2$ subsite is surrounded by $\beta$-ribbon, $\alpha 2$ helix and the loop between $\beta F 1$ and $\beta \mathrm{G} 1$ strands, which is a pocket with enough size to accommodate various residues. However, it is seemed that the superposition doesn't provide much clue on the direct interaction between P2 and S2. In the reference structure, only CVA $3 \mathrm{C}^{\text {pro }}$ shows one interaction position, one backbone H-bond formed between S128 and P2. But in SVA 3C ${ }^{\text {pro }}$, the corresponding position is too far to interact with P2. 
The P1 residue can be superimposed into the pocket formed by 155-160aa and 178-180aa of SVA $3 C^{\text {pro }}$. H48 within $\alpha 2$ helix, T155, G158, W159, C160, H178 and S179 are seemed possible to form H-bonds with both main chain and side chain of P1 residue. The above positions are all conserved among picornaviral $3 \mathrm{C}^{\mathrm{pro}}$, except for S179. In Enterovirus, it is a hydrophobic amino acid residue valine or isoleucine interact with P1 backbone instead of a serine with hydrophilicity. Moreover, the difference within 158-162aa is notable. 159-162aa is corresponding to the G-X-C/S-G-G motif, which is highly conserved in picornaviral $3 C^{\text {pro }}$. In SVA $3 C^{\text {pro }}$, the G162 is mutated into serine, and forms extra H-bonds with S119 from $\beta A 2$ strand and neighboring W159. What's more, an $\alpha$ helix was formed with the participation of G158 and W159, which is not observed in other picornaviral $3 \mathrm{C}^{\mathrm{pro}}$. The extra $\alpha$ helix drags 156-158aa closer to the substrate binding groove. The large side chain from K157 formed an fingerlike projection over S1 and S2 subsites with its tips pointing to the $\beta$-ribbon. All these transformation makes the S1 subsite of SVA $3 C^{\text {pro }}$ smaller than the other.

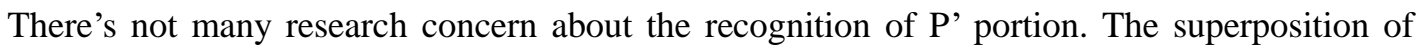
SVA $3 C^{\text {pro }}$ onto the FMDV $3 C^{\text {pro }}$-APAKELLNF peptide co-crystal structure implies that G30, L31, T32, Q33, N52 might associate with the forming of S' pockets. According to the measurement result (table 3), SVA S1' pocket is smaller than FMDV, while S2' and S3' are approximately equivalent, suggesting SVA $3 \mathrm{C}^{\text {pro }}$ may have higher specifity to sequence variance of $\mathrm{P}^{\prime}$ portion. As

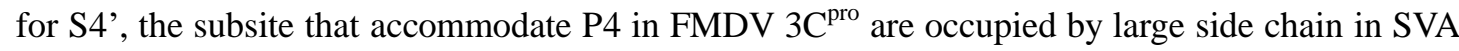
$3 \mathrm{C}^{\mathrm{pro}}$, which causes in the superimpose result $\mathrm{P} 4$ pierces into the surface of SVA $3 \mathrm{C}^{\mathrm{pro}}$ (Fig. 6A ). 


\section{Discussion}

We report here the first structural insights into SVA $3 C^{\text {pro }}$. Our result reveals several unexpected differences of the structure between SVA $3 C^{\text {pro }}$ and other known picornaviral $3 C^{\text {pro }}$. The main difference is SVA $3 C^{\text {pro }}$ adopts a wider S4 pocket and smaller S1, S1' pockets.

A previous research on EV71 and CVA16(Lu et al., 2011) suggested that mutate small amino acid residue into larger one at $\mathrm{P} 4$ and $\mathrm{P} 1$ ' positions could dramatically reduce the rate of peptide cleavage. They explained this phenomenon by observing the limited S4 subsite in both EV71 and CVA16 $3 \mathrm{C}^{\mathrm{pro}} \mathrm{s}$, and reckoned $\mathrm{S} 1$ ' subsite as a small subsite too. According to their study, the protease efficiency is restrict by limitied $\mathrm{P} 4$ and $\mathrm{S} 1$ ' pocket when come across with larger amino acid side chain within cleavage sequence. In this regard, we propose a conjecture about the relationship between the structure of the binding groove and the cleavage efficiency. The capacity of the binding groove might be related to the cleavage efficiency. Although small pockets may be able to accommodate large side chain amino acids due to they can only form $\mathrm{H}$ - bonds with the peptide backbone, but their cleavage efficiency may still significant reduce when encountering larger side chain amino acids. This influence might be weakened by enlarged pocket or more flexible pocket. The latter situation can be achieved by steric hindrance changes, lower steric hindrance can enhance the flexibility of residues nearby binding pocket, and finally result in the increasing of the pockets' accommodation ability. It should be noted that too wide binding grooves could also slightly impaired the efficiency probably because of $\mathrm{P}$ residue is bound less tightly in the enlarge pocket(Zunszain et al., 2010).

According to Lu G's paper(Lu et al., 2011), CVA16 3C ${ }^{\text {pro }}$ could cleave its own structural protein as well as structural protein of EV71 with surprisingly higher efficiency than EV71. We look back into the structures of EV71 $3 C^{\text {pro }}$ and CVA16 $3 C^{\text {pro }}$, the result seems to partially confirmed our conjecture of the relationship between $\mathrm{S} 4$ subsite's structure and cleavage efficiency: two residues within $3 \mathrm{C}^{\text {pro }}$ participating in forming backbone H-bond with substrate P4 position, N126 and S128, have same orientation but different coordinates in EV71 and CVA16.

Further study still need to be carried out in order to demonstrate if the above rules hold between SVA and FMDV, as well as other picornavirus. If the accomodation ability of bindng pocket is truly relevant to cleavage efficiency, taking FMDV as an example: SVA $3 \mathrm{C}^{\text {pro }}$ has a wider $\mathrm{S} 4$ pockets than FMDV, while $\mathrm{P} 4$ is a small amino acid residue in FMDV poly-protein cleavage site (Fig. 2A), so SVA may show higher cutting efficiency when applying to cut FMDV poly-protein. On the other hand, in FMDV poly-protein, the amino acids occupying subsites $\mathrm{S} 1$ and $\mathrm{S} 1$ ' are usually glutamic acid/glutamine and glycine/threonine (Fig. 2A), those side chains size are similar to those in SVA. Therefore, although the S1, S1' pockets are smaller in SVA $3 \mathrm{C}^{\text {pro }}$, it may not significantly influence the cleavage efficiency when cutting FMDV poly-protein.

In summary, the structural studies on $3 \mathrm{C}^{\mathrm{pro}}$ could help to reveal the cleavage mechanism of $3 \mathrm{C}^{\mathrm{pro}}$ in picornaviruses, and provide theoretical basis for the subsequent design of VLP vaccines. 


\section{Materials and Methods}

Bioinformatics analysis. The phylogenetic analysis of picornaviral $3 \mathrm{C}^{\text {pro }}$ was carried out by MEGA 7 (Kumar et al., 2016). And the $3 C^{\text {pro, }}$ s cleavage specificities is analyzed by ClustalW (Larkin et al., 2007), Espript (Robert and Gouet, 2014) and kpLogo (Wu and Bartel, 2017). All sequences were derived from GenBank, accession numbers are as follows: Poliovirus (PV), CAA24465; Hepatitis A virus (HAV), AAA45466; Human TMEV-like cardiovirus (HTCV),ACB29695; Human Coxsackievirus A16 (CVA16), ACV33370; Coxsackievirus B3 (CVB3), AAA42931; Human rhinovirus A (HRV-A), ACK37367; Human rhinovirus B (HRV-B), BAA00168; Human rhinovirus C (HRV-C), ACZ67658; Enterovirus D68 (EV68), ABL61317; Human enterovirus 71 (EV71), ACY00662; Foot-and-mouth disease virus - type A (FMDV-A), AZS18886; Foot-and-mouth disease virus - type O (FMDV-O), AAG45408; Foot-and-mouth disease virus - type SAT2 (FMDV-SAT2), AAQ11227; Equine rhinitis B virus (ERBV), CAA65615; Porcine teschovirus (PTV), CAB40546; Bovine rhinitis A virus (BRAV), AKA20760; Porcine sapelovirus (PSV), AAM33242; Encephalomyocarditis virus (EMCV), AAA43037.

Protein production. The original viral strain selected in this study was Seneca valley virus isolate SVV-001 (GenBank accession no. DQ641257). The gene for 3C $\mathrm{C}^{\text {pro }}$ was amplified using the primers 3C-forward (5'-GGAATTCCATATGCAGCCGAACGTAGATATGG-3') and 3C-reverse (5'-CCGCTCGAGTTACTGCATAGTGGCCAG-3'). The gene was then subcloned into pET-28a via NdeI and XhoI restriction sites, which generated a protein gene followed by an $\mathrm{N}$-teriminal poly-histidine tag coding sequence. The construct was verified by DNA sequencing. The plasmids were then transformed into Escherichia coli BL21 (DE3) competent cells. The expression of 3C ${ }^{\text {pro }}$ was induced by $0.5 \mathrm{mM}$ IPTG at $18^{\circ} \mathrm{C}$ for $16-20 \mathrm{hr}$.

Protein purification and crystallization. The expressed protein was initially purified in an ÄKTA Start system (GE Healthcare) by immobilized metal-affinity chromatography (IMAC) on a $5 \mathrm{ml}$ HisTrap HP column (GE Healthcare). And further purified by gel filtration on a Hi Load Superdex 75 column (GE Healthcare). Peak fractions were concentrated to $10 \mathrm{mg} / \mathrm{ml}$ in $20 \mathrm{mM}$ HEPES, pH 7.5, containing $150 \mathrm{mM} \mathrm{NaCl}$. Crystals were obtained by sitting drop vapor diffusion experiments at $18{ }^{\circ} \mathrm{C}$ with commercial screening kits (HamptonReserch). Optimization of crystallization conditions was performed manually by hanging drop vapor diffusion experiments at $4{ }^{\circ} \mathrm{C}$ and $18{ }^{\circ} \mathrm{C}$.

Data collection and structure determination. Crystals were transferred to a 1:1 mix of reservoir solution and cryo-protectant solution and frozen in a stream of $\mathrm{N}_{2}$ gas at $100 \mathrm{~K}$ immediately prior to data collection. The data collection was performed at the Shanghai Synchrotron Radiation Facility (SSRF) using beamline BL17U at a wavelength of $1.5418 \AA$ (Shanghai, China) (Wang et al., 2018; Wang et al., 2016). The collected intensities were indexed, integrated, corrected for absorption, scaled, and merged using the HKL2000 package (Otwinowski and Minor, 1997).X-ray diffraction data were processed with CCP4 program suite(Collaborative Computational Project, 1994). The crystals structures were determined by molecular-replacement method by using FMDV 3C ${ }^{\text {pro }}$ structure (Protein Data Bank code 2BHG) as the search model (Lebedev et al., 2008; McCoy, 2007). The initial model was obtained by Phaser MR, manual model adjustment and subsequently refinement were performed with COOT and Refmac5 
bioRxiv preprint doi: https://doi.org/10.1101/2020.02.21.959106; this version posted February 21, 2020. The copyright holder for this preprint (which was not certified by peer review) is the author/funder. All rights reserved. No reuse allowed without permission.

respectively(Emsley and Cowtan, 2004). 


\section{Reference}

Belsham, G., and Bøtner, A. (2015). Use of recombinant capsid proteins in the development of a vaccine against the foot-and-mouth disease virus. Virus Adaptation and Treatment 7, 11-23.

Berger, A., and Schechter, I. (1970). Mapping the active site of papain with the aid of peptide substrates and inhibitors. Philos Trans R Soc Lond B Biol Sci 257, 249-264.

Birtley, J.R., Knox, S.R., Jaulent, A.M., Brick, P., Leatherbarrow, R.J., and Curry, S. (2005). Crystal structure of foot-and-mouth disease virus $3 \mathrm{C}$ protease. New insights into catalytic mechanism and cleavage specificity. J Biol Chem 280, 11520-11527.

Collaborative Computational Project, N. (1994). The CCP4 suite: programs for protein crystallography. Acta Crystallogr D Biol Crystallogr 50, 760-763.

Cui, S., Wang, J., Fan, T., Qin, B., Guo, L., Lei, X., Wang, J., Wang, M., and Jin, Q. (2011). Crystal structure of human enterovirus 71 3C protease. J Mol Biol 408, 449-461.

Curry, S., Roqué-Rosell, N., Zunszain, P.A., and Leatherbarrow, R.J. (2007). Foot-and-mouth disease virus $3 \mathrm{C}$ protease: recent structural and functional insights into an antiviral target. Int $\mathrm{J}$ Biochem Cell Biol 39, 1-6.

Emsley, P., and Cowtan, K. (2004). Coot: model-building tools for molecular graphics. Acta Crystallogr D Biol Crystallogr 60, 2126-2132.

Kumar, S., Stecher, G., and Tamura, K. (2016). MEGA7: Molecular Evolutionary Genetics Analysis Version 7.0 for Bigger Datasets. Mol Biol Evol 33, 1870-1874.

Larkin, M.A., Blackshields, G., Brown, N.P., Chenna, R., McGettigan, P.A., McWilliam, H., Valentin, F., Wallace, I.M., Wilm, A., Lopez, R., et al. (2007). Clustal W and Clustal X version 2.0. Bioinformatics 23, 2947-2948.

Lebedev, A.A., Vagin, A.A., and Murshudov, G.N. (2008). Model preparation in MOLREP and examples of model improvement using X-ray data. Acta Crystallogr D Biol Crystallogr 64, 33-39.

Leong, L.E., Walker, P.A., and Porter, A.G. (1993). Human rhinovirus-14 protease 3C (3Cpro) binds specifically to the 5'-noncoding region of the viral RNA. Evidence that 3Cpro has different domains for the RNA binding and proteolytic activities. J Biol Chem 268, 25735-25739.

Lu, G., Qi, J., Chen, Z., Xu, X., Gao, F., Lin, D., Qian, W., Liu, H., Jiang, H., Yan, J., et al. (2011). Enterovirus 71 and coxsackievirus A16 3C proteases: binding to rupintrivir and their substrates and anti-hand, foot, and mouth disease virus drug design. J Virol 85, 10319-10331.

Matthews, D.A., Smith, W.W., Ferre, R.A., Condon, B., Budahazi, G., Sisson, W., Villafranca, J.E., Janson, C.A., McElroy, H.E., Gribskov, C.L., et al. (1994). Structure of human rhinovirus 3C protease reveals a trypsin-like polypeptide fold, RNA-binding site, and means for cleaving precursor polyprotein. Cell 77, 761-771.

McCoy, A.J. (2007). Solving structures of protein complexes by molecular replacement with Phaser. Acta Crystallogr D Biol Crystallogr 63, 32-41.

Mosimann, S.C., Cherney, M.M., Sia, S., Plotch, S., and James, M.N. (1997). Refined X-ray crystallographic structure of the poliovirus 3C gene product. J Mol Biol 273, 1032-1047.

Otwinowski, Z., and Minor, W. (1997). Processing of X-ray diffraction data collected in oscillation mode. Methods Enzymol 276, 307-326.

Polacek, C., Gullberg, M., Li, J., and Belsham, G.J. (2013). Low levels of foot-and-mouth disease virus $3 \mathrm{C}$ protease expression are required to achieve optimal capsid protein expression and processing in mammalian cells. J Gen Virol 94, 1249-1258.

Porta, C., Xu, X., Loureiro, S., Paramasivam, S., Ren, J., Al-Khalil, T., Burman, A., Jackson, T., 
Belsham, G.J., Curry, S., et al. (2013). Efficient production of foot-and-mouth disease virus empty capsids in insect cells following down regulation of $3 \mathrm{C}$ protease activity. J Virol Methods 187 , 406-412.

Robert, X., and Gouet, P. (2014). Deciphering key features in protein structures with the new ENDscript server. Nucleic Acids Res 42, W320-324.

Walker, P.A., Leong, L.E., and Porter, A.G. (1995). Sequence and structural determinants of the interaction between the 5'-noncoding region of picornavirus RNA and rhinovirus protease $3 \mathrm{C}$. J Biol Chem 270, 14510-14516.

Wang, Q.-S., Zhang, K.-H., Cui, Y., Wang, Z.-J., Pan, Q.-Y., Liu, K., Sun, B., Zhou, H., Li, M.-J., Xu, Q., et al. (2018). Upgrade of macromolecular crystallography beamline BL17U1 at SSRF. Nuclear Science and Techniques 29, 68.

Wang, Z., Pan, Q., Yang, L., Zhou, H., Xu, C., Yu, F., Wang, Q., Huang, S., and He, J. (2016). Automatic crystal centring procedure at the SSRF macromolecular crystallography beamline. J Synchrotron Radiat 23, 1323-1332.

Wu, X., and Bartel, D.P. (2017). kpLogo: positional k-mer analysis reveals hidden specificity in biological sequences. Nucleic Acids Res 45, W534-W538.

Zhang, X., Zhu, Z., Yang, F., Cao, W., Tian, H., Zhang, K., Zheng, H., and Liu, X. (2018). Review of Seneca Valley Virus: A Call for Increased Surveillance and Research. Front Microbiol 9, 940.

Zunszain, P.A., Knox, S.R., Sweeney, T.R., Yang, J., Roque-Rosell, N., Belsham, G.J., Leatherbarrow, R.J., and Curry, S. (2010). Insights into cleavage specificity from the crystal structure of foot-and-mouth disease virus 3C protease complexed with a peptide substrate. J Mol Biol 395, 375-389. 
Table 1. Data collection and refinement statistics

\begin{tabular}{ll}
\hline Data collection and refinement parameter & Result \\
\hline Diffraction data & \\
Space group & $\mathrm{P} 12_{1} 1$ \\
Cell dimensions & \\
$\quad \mathrm{a}, \mathrm{b}, \mathrm{c}(\AA)$ & $45.91,57.6,74.78$ \\
$\alpha, \beta, \gamma\left({ }^{\circ}\right)$ & $90,100.26,90$ \\
Resolution range $(\AA)$ & $27.57-1.85$ \\
No. of unique reflections & 32921 \\
Multiplicity & $6.1(6.3)$ \\
Completeness $(\%)$ & $99.9(99.9)$ \\
$\mathrm{I} / \sigma_{\mathrm{I}}$ & $11.6(8.3)$ \\
$\mathrm{R}_{\text {merge }}(\%)$ & $11.5(16.7)$ \\
& \\
Model refiement & \\
No. of atoms & \\
Protein & 1552 \\
Water & 101 \\
$\mathrm{R}_{\text {factor }}(\%)$ & 18.43 \\
$\mathrm{R}_{\text {free }}(\%)$ & 23.55 \\
RMSD & \\
Bond lengths $(\AA)$ & 0.0104 \\
Angles $\left({ }^{\circ}\right)$ & 1.5726 \\
Average $\mathrm{B}-$ factor $\left(\AA^{2}\right)$ & 11.184 \\
Ramachandran plot & \\
Most favored regions $(\%)$ & 95.07 \\
In allowed regions $(\%)$ & 4.93 \\
Disallowed region $(\%)$ & 0 \\
\hline
\end{tabular}


Table 2. Alignment of SVA 3Cpro and other known $3 C^{\text {pro }} \mathrm{s}$

\begin{tabular}{rcccc}
\hline \hline & $\begin{array}{c}\text { N-terminus } \\
\text { RMSD(A) }\end{array}$ & $\begin{array}{c}\text { Loop } \\
\text { RMSD }(\AA)\end{array}$ & $\begin{array}{c}\text { C-terminus } \\
\text { RMSD }(\AA)\end{array}$ & $\begin{array}{c}\text { Overall } \\
\text { RMSD }(\AA)\end{array}$ \\
\hline Without substrate & & & & \\
SVA-CVA16 (3SJ8) & 1.641 & 2.582 & 1.492 & 1.703 \\
SVA-CVB3 (2ZU1) & 2.135 & 2.160 & 1.654 & 1.927 \\
SVA-EV68 (3ZV8) & 2.024 & 2.254 & 1.635 & 1.876 \\
SVA-EV71 (3OSY) & 1.683 & 2.514 & 1.682 & 1.798 \\
SVA-FMDV A10 (2BHG) & 1.611 & 1.958 & 1.250 & 1.515 \\
SVA-FMDV SAT2 (5HM2) & 1.657 & 2.338 & 1.335 & 1.607 \\
SVA-HAV (1HAV) & 2.180 & 2.019 & 1.689 & 1.944 \\
With substrate & & & & \\
SVA-PV (4DCD) & 1.759 & 2.200 & 1.445 & 1.680 \\
SVA- CVA16 (3SJ9) & 1.731 & 2.654 & 1.466 & 1.747 \\
SVA-CVB3 (2ZU3) & 2.029 & 2.093 & 1.599 & 1.829 \\
SVA-EV68 (3ZVA) & 2.064 & 2.236 & 1.502 & 1.893 \\
SVA-EV71 (3SJO) & 1.682 & 2.568 & 1.509 & 1.731 \\
SVA-HRV2 (1CQQ) & 1.855 & 2.415 & 1.495 & 1.768 \\
SVA-FMDV A10 (2WV5) & 1.568 & 1.973 & 1.100 & 1.424 \\
SVA- HAV(2HAL) & 2.163 & 2.037 & 1.386 & 1.828 \\
\hline \hline
\end{tabular}

Table 3. Width of the $\mathrm{S}$ subsite within picornaviral $3 \mathrm{C}^{\mathrm{pro}} \mathrm{S}$

\begin{tabular}{lll}
\hline \hline & SVA & FMDV \\
\hline S4 & $11.1 \AA$ & $9.2 \AA$ \\
S3 ${ }^{*}$ & NA & NA \\
S2 & $9.1 \AA$ & $9.5 \AA$ \\
S1 & $6.9 \AA$ & $7.8 \AA$ \\
S1 ${ }^{\prime}$ & $10.9 \AA$ & $12 \AA$ \\
$\mathrm{S}^{\prime}$ & $8.3 \AA$ & $8.6 \AA$ \\
$\mathrm{S}^{\prime}$ & $7.0 \AA$ & $6.9 \AA$ \\
$\mathrm{S}^{\prime}{ }^{*}$ & $\mathrm{NA}$ & $\mathrm{NA}$ \\
\hline \hline
\end{tabular}

* S3 and S4' shows no significant pocket structure. 


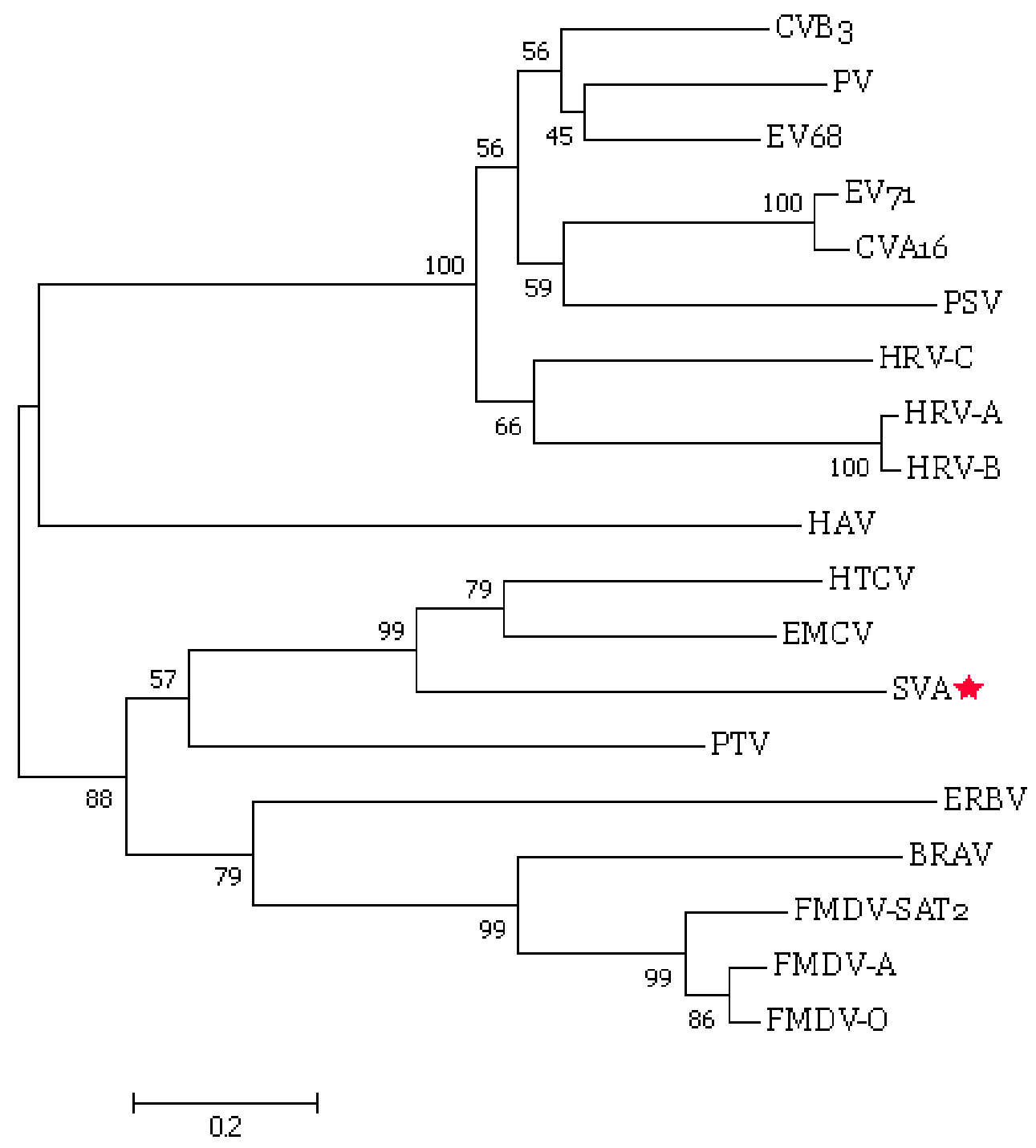

Figure 1. Phylogenetic comparison of the picrnaviral $3 C^{\text {pro }}$ sequences. Midpoint-rooted Neighbor-joining tree were produced in MEGA 7 (Kumar et al., 2016) using the Poisson model. The number of bootstrap replicates were set as 2000. Bootstrap values are given at the nodes. 
A

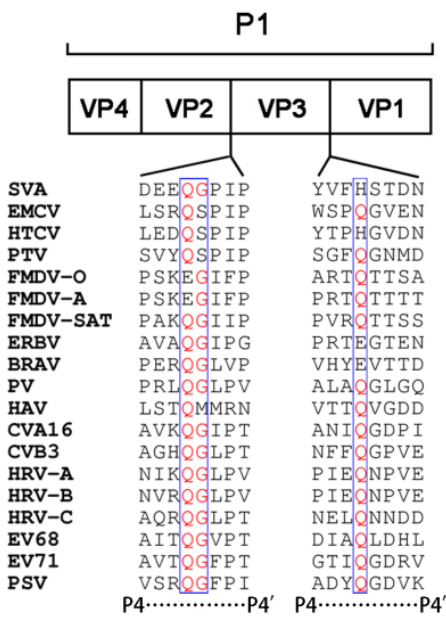

중

정

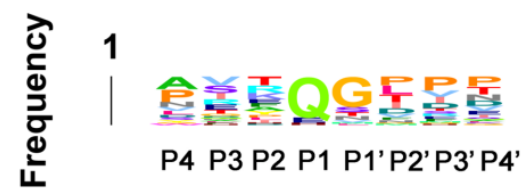

Figure 2. (A) Overview of P1 region organization within the picornaviral polyproteins. The two cleavage junction sites that are to be processed by $3 \mathrm{C}^{\text {pro }}$ are indicated, with each joining sequence spanning from the P4 to P4' residues listed below the schematic. (B) Sequence logo of VP0-VP3 cleavage site. (C) Sequence logo of VP3-VP1 cleavage site. (D) Sequence logo of both cleavage sites.

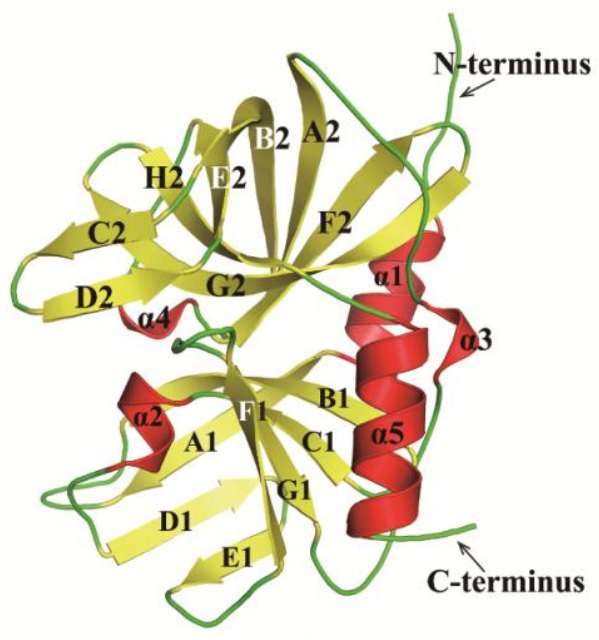

A

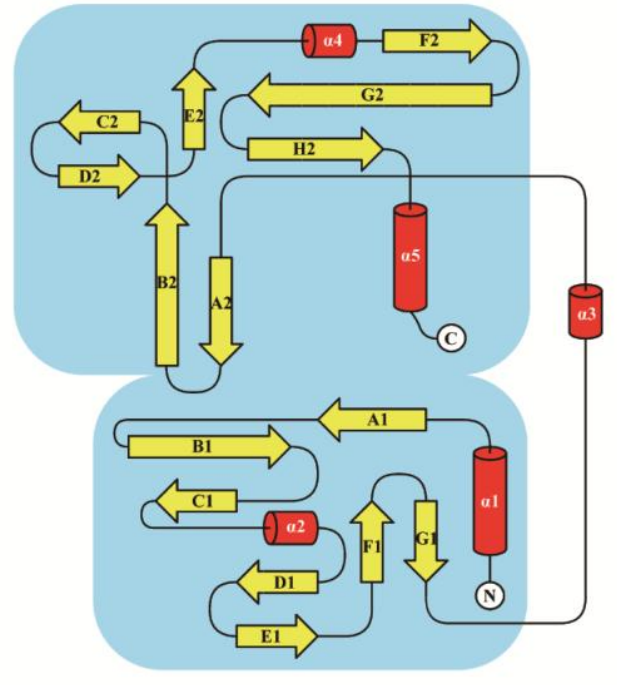

B

Figure 3. The overall structure of SVA $3 C^{\text {pro }}$. (A) Cartoon representation of the structure of SVA $3 C^{\text {pro }}$. (B) Topology diagram of SVA $3 C^{\text {pro }}$. 


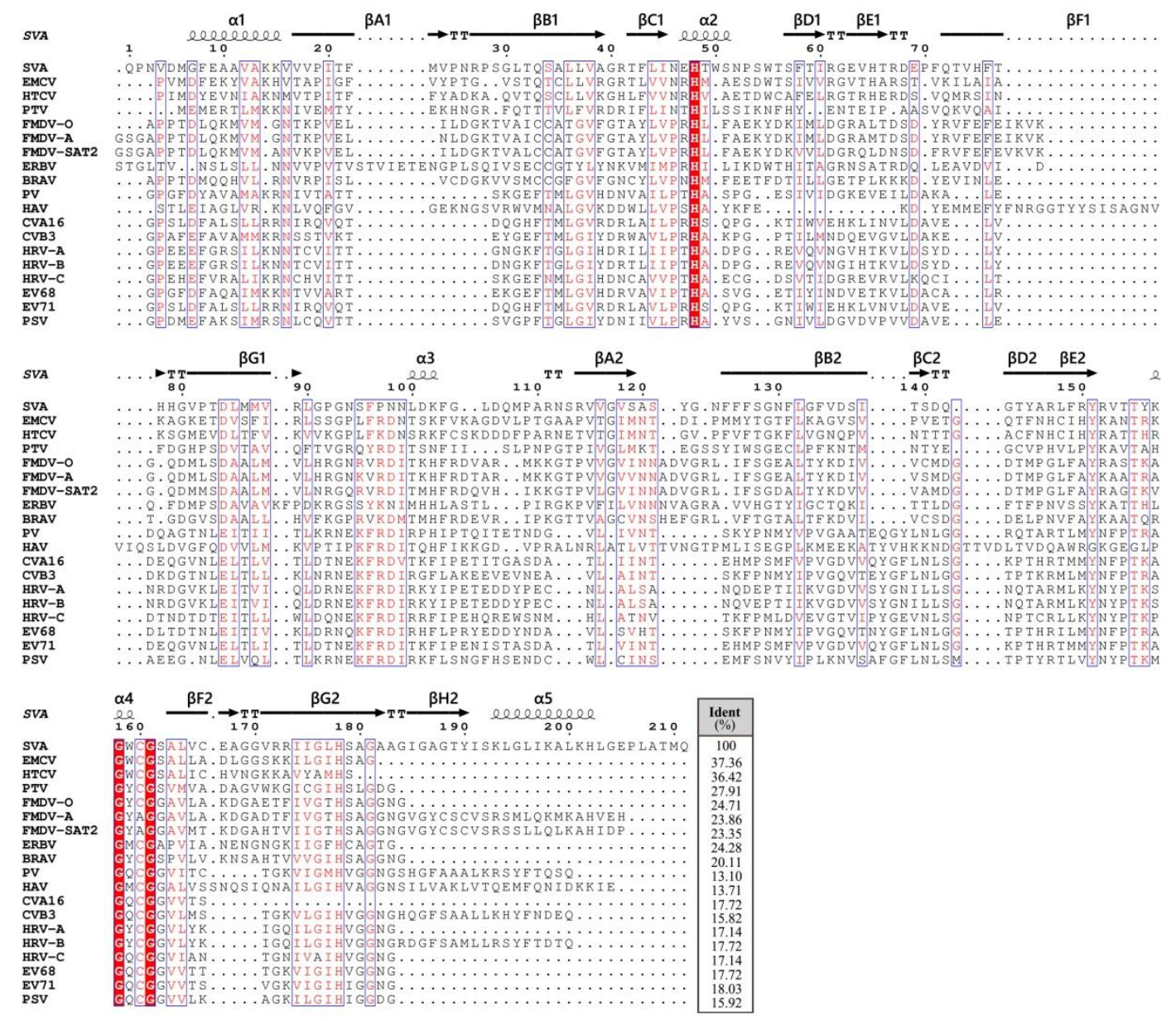

Figure 4. Structure based multiple-sequence alignment of the $3 C^{\text {pro }}$ from common picornaviruses. Invariant residues in $3 \mathrm{C}$ pro are highlighted with red background; conserved residues are shown in red font. 


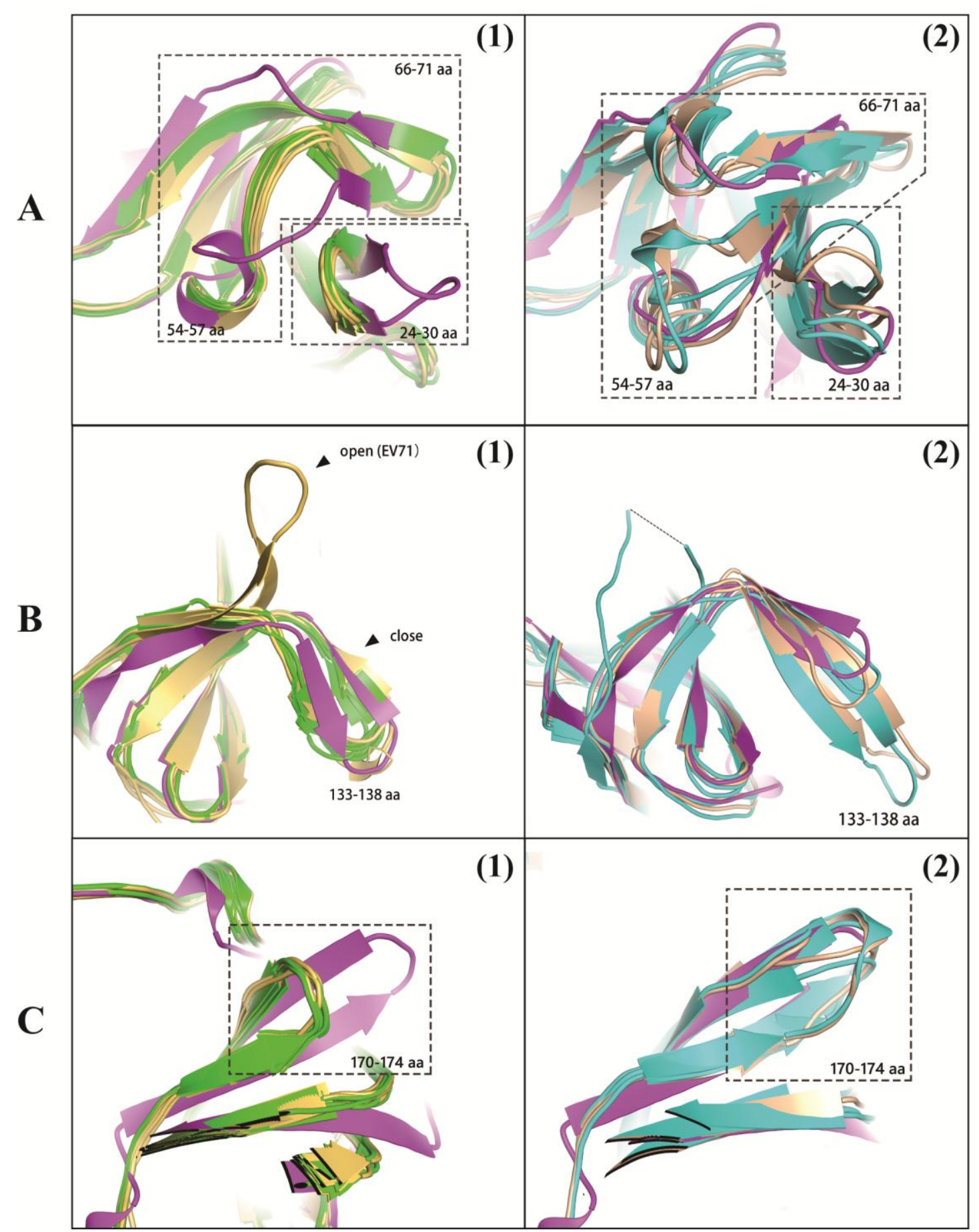

Figure 5. Superposition of SVA $3 C^{\text {pro }}$ (magneta) on other known $3 C^{\text {pro }}$ s. The superposition is separated into two groups, in order to exhibt major differences legibly. Group 1 (without substrate - yellow, with substrate - green) including enterovirus and rhinovirus; group 2 (without substrate - cyan, with substrate - wheat) including FMDV and HAV. (A) Cartoon representation of major difference within 24-30aa, 54-57aa and 66-71 aa. (B) Cartoon representation of major difference within 133-138 aa. (C) Cartoon representation of major difference within 170-174 aa. 
bioRxiv preprint doi: https://doi.org/10.1101/2020.02.21.959106; this version posted February 21, 2020. The copyright holder for this preprint (which was not certified by peer review) is the author/funder. All rights reserved. No reuse allowed without permission.
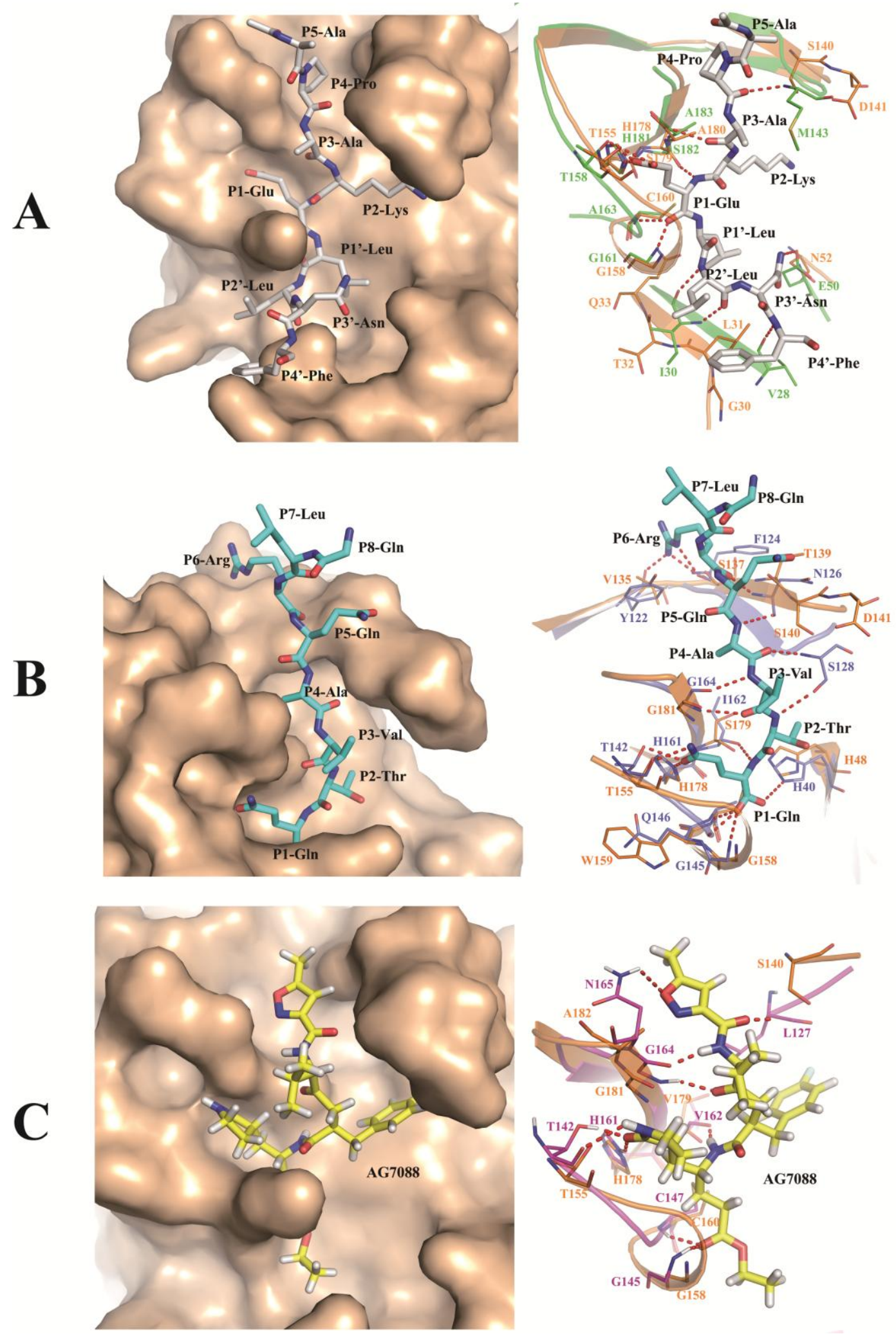

Figure 6. Prediction on residues of SVA 3Cpro directly involve in substrate binding. (A) Superimpose the SVA $3 \mathrm{C}^{\text {pro }}$ (orange) onto the FMDV 3Cpro-APAKELLNF peptide co-crystal structure. The peptide is shown as white, and the residues interact with the substrate is shown as green lines. (B) Superimpose the SVA $3 C^{\text {pro }}$ onto the CVA 3Cpro-GLRQAVTQ peptide co-crystal structure. The peptide is shown as cyan, and the residues interact with the substrate is shown as light blue lines. (C) Superimpose the SVA 3C ${ }^{\text {pro }}$ onto the HRV 3Cpro-AG7088 co-crystal 
bioRxiv preprint doi: https://doi.org/10.1101/2020.02.21.959106; this version posted February 21, 2020. The copyright holder for this preprint (which was not certified by peer review) is the author/funder. All rights reserved. No reuse allowed without permission.

structure. The inhibitor is shown as yellow, and the residues interact with the substrate is shown as magneta lines. 